\title{
ASSURING THE SDN SECURITY BY MODELLING AND COMPARING SDN PROPOSED TOPOLOGIES USING PETRI NETS
}

\author{
Ali Ameen, ORCID ID: 0000-0002-5451-8257 \\ Technical University of Moldova, 168 Stefan cel Mare Blvd., MD-2004 Chisinau, Republic of Moldova \\ Corresponding author: Ali Ameen, alisalmanhussein@yahoo.com
}

Received: 09. 04. 2021

Accepted: 11. 12. 2021

\begin{abstract}
The soaring number of applications for autonomous systems in different aspects like air, sea, and space is creating the need for new methodologies and architectures' technologies to consolidate the verification of system-level and system-of-systems level. The implementation of cybersecurity standards and software is critical to supporting infrastructure. This article discusses some security issues regarding autonomous systems' computer networks. It proposes the usage of Software-Defined Networks (SDN) technologies as a solution, after providing better security in SDN environment through the usage of the HYDRA framework and the usage of multiple controllers in specific topologies to ensure the security of SDN in precise and to ensure the security of the autonomous systems' computer networks in general as well. We propose a framework that contains 3 different types of controllers' topologies and each topology can use 4 algorithms, HYDRA, VPN, Double RSA, and least but not last comes blockchain technology which is the core of our security.
\end{abstract}

Keywords: autonomous, systems, computer, network, security, SDN, technology, controller, topology, algorithm.

Rezumat. Numărul tot mai mare de aplicații pentru sisteme autonome în diferite aspecte precum aerul, marea și spațiul creează nevoia de noi metodologii și tehnologii de arhitectură pentru a consolida verificarea la nivel de sistem și la nivel de sistem de sisteme. Implementarea standardelor și a software-ului de securitate cibernetică este esențială pentru sprijinirea infrastructurii. Acest articol discută unele probleme de securitate privind rețelele de calculatoare ale sistemelor autonome. Acesta propune utilizarea tehnologiilor SoftwareDefined Networks (SDN) ca soluție, după ce a asigurat o mai bună securitate în mediul SDN prin utilizarea cadrului HYDRA și utilizarea mai multor controlere în topologii specifice pentru a asigura securitatea SDN în mod precis și pentru asigurarea securității rețelelor de calculatoare ale sistemelor autonome și în general. Propunem un cadru care conține 3 tipuri diferite de topologii de controlere și fiecare topologie poate folosi 4 algoritmi, HYDRA, VPN, Double RSA, și cel mai puțin, dar nu ultimul, este tehnologia blockchain care este nucleul securității noastre.

Cuvinte cheie: autonom, sisteme, computer, rețea, securitate, SDN, tehnologie, controler, topologie, algoritm. 


\section{Introduction}

The soaring number of applications for autonomous systems in different aspects like air, sea, and space is creating the need for new methodologies and architectures' technologies to consolidate the verification of system-level and system-of-systems level. The implementation is critical for backing up the cybersecurity standards and software infrastructure. The classical structure of networks has remained unchanged for a long time till the development of the new structure of software-defined network came up starting with its first attempt by Martin Casado which and that model was named Ethane [1]. Most security issues related to the heritage structure and hierarchy could be solved using the new paradigm of SDN but, due to the relative novelty of SDN methodology in managing the computer networks; many other new cyber-security challenges could emerge and with that comes the need to secure that environment to facilitate the transition from the classical network structure to the SDN structure and also there's a need to use risk assessment tools; both software and mathematical ones to determine the level of security risk that lies within a software-defined network environment since that it is useless or with less precision to use the common existing mathematical equations that were originally developed for assessing the security level in classical computer networks' environment.

There are many works and researches to use petri nets to study and analyze the security of computer networks in general like [2]; where they leveraged petri nets to model three defense scenarios the first one was with firewall only, the second was with firewall and Intrusion Detection System IDS and the third defense scenario was with firewall, IDS and a honeypot system. Also, many attempts to address the security issues in SDN in precise have been conducted including modeling the problems mathematically and graphically; the usage of petri nets was one of the main prominent methodologies used for that purpose [3] where they try in this work to model the SDN structure and model it under Denial of Service (DoS) attack but, not on the proposed topologies by this article. Also, in [4] the researchers tried to verify and give a general analysis for the security situation in the software-defined network environment that contains two switches and one controller; without the need to discuss the impact of a (DoS/DDoS) attack and again the modeled topology differs from those proposed in this article.

This article mainly discusses the topic of enhancing and ensuring the security of software-defined networks by suggesting several algorithms working together as a framework and by suggesting three different SDN controllers' topologies for the controllers of software-defined networks to overcome some cyber-security issues and to ensure the network reliability against various cyber-threats specifically, the threats of DoS/ DDoS [5]. We have discussed comparison. Also, this article presents a defense factor equation which is a new equation to assess the level of security risk in any SDN environment especially if it was based on any of the three presented structures in this research; since that most works haven't discuss that matter and even when they did, it wasn't based on the topologies proposed here and not discussing the previously mentioned attacks in precise as well and from that stems the importance and novelty of the research and this article that discusses it. And to discuss that specific matter, this article proposes the usage of petri nets modeling paradigm [9] to get simulation results to gain a better reading for the situation of the interaction between controllers and their networks and how would they react under the effect of DoS/DDoS attacks. In the section 2; we'll demonstrate briefly some of the main algorithms and methods proposed to form the framework that will be as a security solution each of them separately in 
other articles [6 - 8] but here we'll gather them all for the purposes of to patch different security breaches, in section 3 we'll talk about the main idea of the paper which is the Petri Nets modelling for the suggested SDN controllers' topologies to derive an equation that could be used as a mathematical tool to measure the defense capabilities of the software-defined networks that leverage our proposed models against different cyber-threats; especially the DoS/DDoS attacks. Least but not last there will be a conclusion for the whole paper in section 4.

\section{Integrated algorithms into the proposed framework}

There are few algorithms proposed in the research and we'll describe them briefly here in this paper. Those algorithms work together to form the whole framework that will be installed on top of the management layer or the application plane which.

\section{Algorithms' suite integrated in HYDRA framework.}

The framework will be installed upon the management plane; it is based on some algorithms and methodologies like the counter measurement attack against the perpetrator. Also it contains other technologies and algorithms as mentioned below.

\section{Secured channel of VPN algorithm.}

The secured channel provided by Virtual Private Network (VPN) technology has a great deal of security that it provides; its Internet Protocol Security (IPsec) algorithm can be leveraged to ensure the security in the communication channel between SDN controllers that is called the east-westbound API [10].

\section{Cryptography of Double RSA algorithm.}

The Rivest-Shamir-Adelman (RSA) algorithm is well known for its robustness in securing computer networks' communication till this day. This algorithm or methodology mainly consists of four stages, which are key generation, key distribution, encryption and decryption. The RSA algorithm contains two main keys' categories they are the public key and the private key. The stages of key generation are [11]:

1. The generation of two large prime numbers let us name them $p$ and $q$.

2. The computation of $n=p * q$.

3. The computation of $z=(p-1) *(q-1)$.

4. Computation of the $\bmod z=v$.

5. Selecting a prime number to $z$ and let's name it as $x$.

6. Figure out the value of e. then we put: $e^{*} x=1 \bmod z$ that means that: $e^{*} x=1^{*} v$.

7. Now let public key be $(n, e)$

8. Now let private key be $(n, x)$.

It is proposed by this research to use it but in a full duplex-like way as shown in Figure 1.

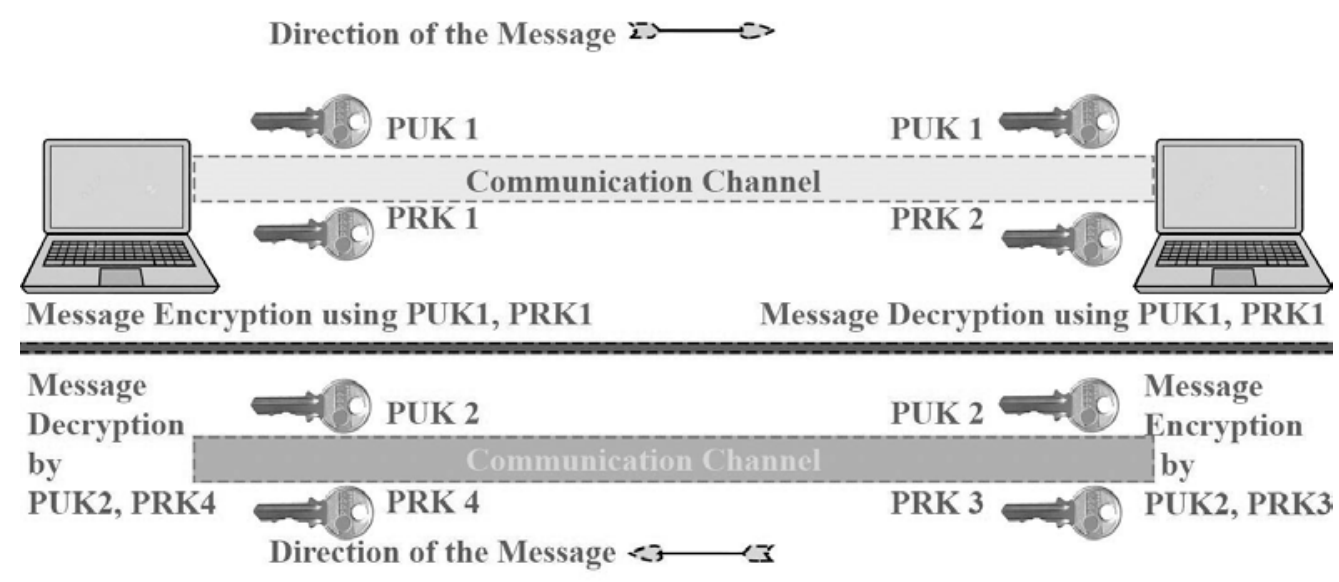

Figure 1. Double RSA. 
Where there will be two public keys instead of one and four private keys instead of two creating to channels or tunnels for every node; where one of them will be used for sending information and the other one for reception.

\section{Distributed ledger concept of Blockchain algorithm.}

Blockchain is a promising technology and we have published an essay about that [12], it is a public ledger with a distributed feature that behaves like a log which could keep track of all transactions in a chronological way, it is secured using a mechanism of appropriate consensus and provides an immutable record [13]. Therefore, it is possible to say that the blockchain could be considered as a decentralized ledger for all peer-to-peer network's transactions. With blockchain, participants could conduct transactions' confirmation without any need for a controlling central authority. Potential usage applications could be trade contracts, voting, fund transfers, etc... [14]. The blockchain technology could be exploited for securing network configuration updates exchanged between multiple controllers in SDN structure, but in a different way than it is used in the Marconi protocol [15].

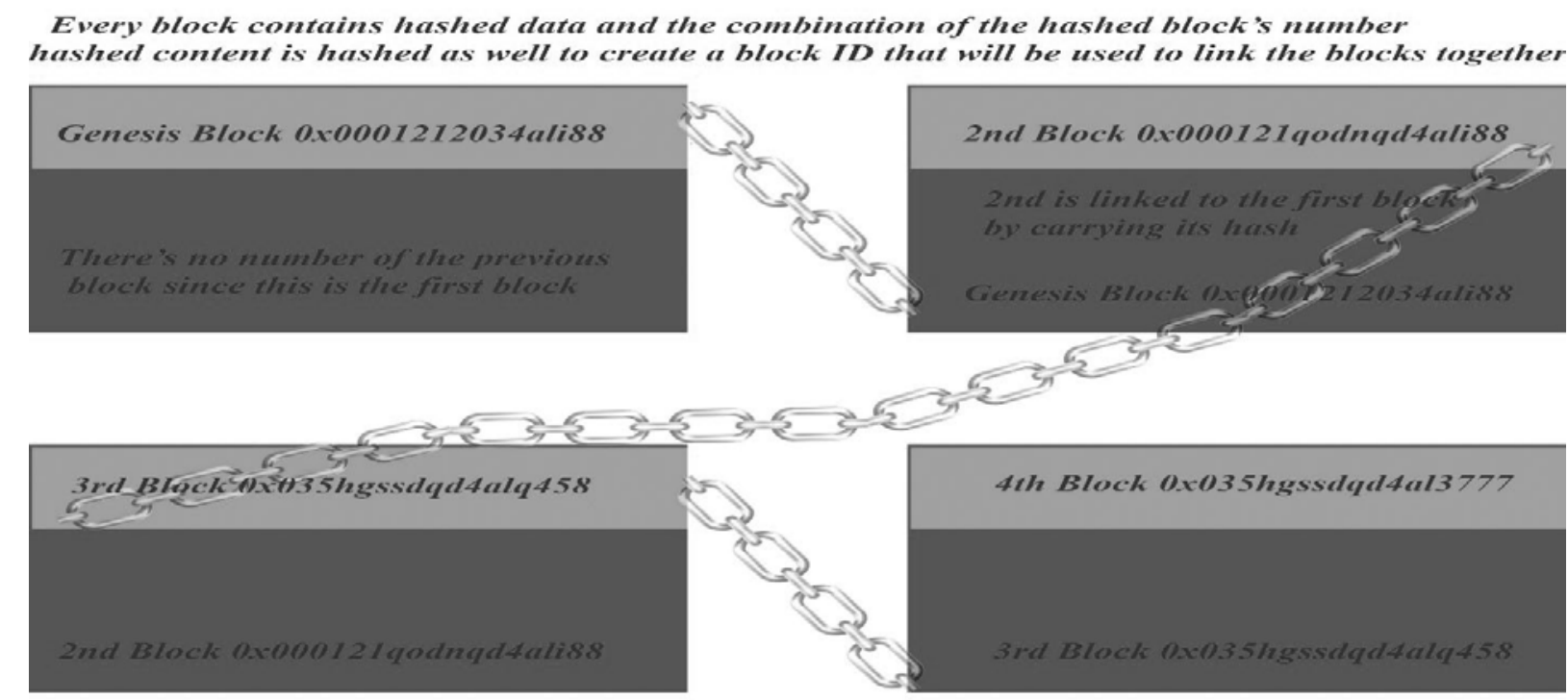

Figure 2. Blockchain.

\section{Modelling the proposed topologies with petri nets}

In the beginning, it is important to give a simple description of the proposed topologies for software-defined networks' environment, that could be a better solution to deal with DoS/DDoS attacks.

Carl Adam Petri was accredited for the invention of Petri nets system and its aim is for describing chemical processes. The Petri Nets is a place/transition (PT) system; and it is a description and a modelling approach for distributed systems. Moreover, it has the definition of a dynamic system of discrete events. Petri Nets are directed bipartite graphs, therefore they're formed of two types of nodes. The two main nodes are places drawn as circles and transitions as bars. Those directed arcs define the direction of procedure and that would figure out which places are pre conditions and which are post conditions. The Petri Net presents a notation of a graphical nature that could be leveraged for diverse purposes and procedures with concurrent implementation. Also, this procedure presents a mathematical definition [3].

There are different types of simulation software that use petri net models so, it is possible to use one of the available choices we have; our choice here is the software named Platform 
Independent Petri Nets Editor (PIPE). One of its main modules that we'll use for our own research is the Generalized Stochastic Petri Nets (GSPN) module that mainly focuses on what we exactly require and that's the amount of tokens that occupy the places which in turn represent the controllers placed in the SDN environment. The tokens here represent the updates or requests to or from the SDN controllers.

Now we are going to demonstrate how the three proposed topologies of controllers work , intercommunicate, and react towards each other. Also, we'll show how they defend themselves to deter different attacks like DoS/DDoS ones by modelling their behavior with petri nets system that uses Markov chains for modeling the behavior of the controllers in each topology. We will use the PIPE software to implement our topologies in petri nets, especially the module of GSPN to gain numerical results. The (GSPN) module, which stands for Generalized Stochastic Petri Nets, is defined as a 6-tuple (P, T, F, W, $\left.M_{0}, \lambda\right)$ [3] such that:

1. $P=\left\{P_{1}, P_{2} \ldots P_{m}\right\}$ describes the places' set $n \geqslant 0$ which are of finite nature.

2. While $T=T_{1} \cap T_{2}, T 1=\left\{t_{1}, t_{2} \ldots t_{m}\right\}$ describes finite timed transitions' group, and each one of those transitions has a delay time of a random nature the delay time is set within the period of enabling or activating and firing. In addition, $T 2=\left\{t_{m+1}, t_{m+2} \ldots t_{n}\right\}$ is the finite immediate transitions' group or set, these transitions can be randomly fired and the delay value is set to zero.

3. Also, $\mathrm{F} \subseteq(\mathrm{PxT}) \cap(\mathrm{TxP})$ is arcs' set; and, there are the inhibitor arcs which can only form places to transitions and disenable the activated conditions.

4. W represents the arcs' weight function: $F \rightarrow\{1,2,3 \ldots\}$.

5. Also, $M_{0}: P \rightarrow\{0,1,2,3 \ldots\}$ represents the initial marking, where $(P \times T)=\varnothing \cap(T \times P)=\varnothing$ [3].

6. $\lambda=\left\{\lambda_{1}, \lambda_{2}, \lambda_{3} \ldots \lambda_{n}\right\}$ represents firing rates' set that represent or describe the timed transitions. Each rate is the average firing times of transition in unit of time [3].

There three topologies presented in our research plus a fourth which is the single-controller Ordinary Topology. We'll compare the three topologies with the ordinary topology to depict the enhancements they have.

\section{Serial topology}

As shown in the figure below, this topology consists of three controllers interacting with each other and communicating as one main controller and two controllers as backup; we should mention that they could be software-oriented or hardware-oriented controlling entities.

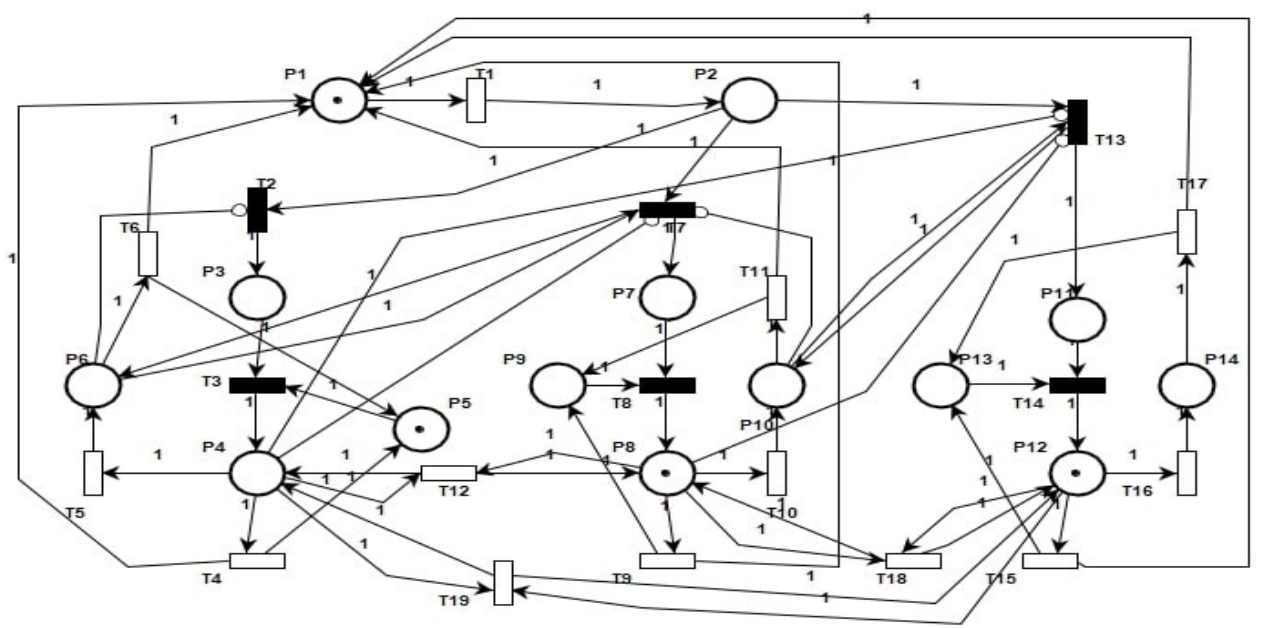

Figure 3. Proposed Serial Topology for Software-Defined Networks. 
In this topology, the selected controller to work as the main controller is working normally and behaving as the decision-making node. This unit administrates the behavior of the network; communicating with the upper layer which is the management layer and with the lower layer which is the data layer and providing management for hosts' requests through the switches of the data plane.

The main controlling unit will send configuration updates and flow tables information of network every period of 10 seconds to the spare controllers to keep them up to date, to keep them as standby network controllers and to be able to continue administering and preserve the network information in case of a cyber-threat that could compromise the previous controlling node.

Again, after compromising the main node in the attack situation; the network management is going to be assigned to the standby controller that has the next number of priority, No. 2 controller for instance is going to be the one in charge in this situation. The next step will be sending a bot to infect the source IP of the attacker afterwards; this source IP of the attacker is going to be added to the blocked IPs in a single direction so the attacker can't send anything anymore just like the access control list (ACL) situation. The compromised controlling node is going to be isolated in the same time. The new elected controller will be the main one after it was the backup one previously. Now this controller administers the environment and exchanges network information with the other last remaining spare controller while the previously main controller is under maintenance. Table 1 provides Petri Nets places' description of the proposed Serial Topology model [6].

Table 1

\section{Description of Places in Serial Topology for SDN}

\begin{tabular}{ll}
\hline Place & Description \\
\hline P1 & $\begin{array}{l}\text { Packets being processed by the main controlling node / the input place } \\
\text { which sends data tokens }\end{array}$ \\
\hline P2 & Servers' selection \\
\hline P3/P7/P11 & Servers' allocation \\
\hline P4/P8/P12 & Active processing in the servers 1,2,3 \\
\hline P5/P9/P13 & Free controllers 1,2,3 \\
\hline P6/P10/P14 & Attack is active \\
\hline
\end{tabular}

The table 2 depicts the serial model transition' description [6].

Table 2

\section{Description of Transitions in Serial Topology for SDN}

\begin{tabular}{ll}
\hline Transition & Description \\
\hline T1 & Task generation i.e., processing of packets \\
\hline T2/T7/T13 & Selection of servers 1, 2, 3 \\
\hline T3/T8/T14 & Allocation of servers \\
\hline T4/T9/T15 & Task processing \\
\hline T5/T10/T16 & Exiting the stage \\
\hline T6/T11/T17 & Restoring the controller \\
\hline T12/T18/T19 & Updating the information and going back to initial stage of controllers \\
\hline
\end{tabular}




\section{Parallel topology.}

This topology consists of three main controllers; which cooperate in managing the whole network as a one entity as if it was one controller. They update each other with the information they have about the contiguous network nodes each controller has as shown in figure 4.

As mentioned before in this topology we'll have 3 controllers as well but, the difference will be in their interaction with each other. The 3 controllers will be working as a whole entity like one brain of 3 parts where they work simultaneously to process switches' requests. They will all behave like the main controller. Each controller will serve switches and prioritize them based on the closest ones to it. Each one of them will send a broadcast update to other remaining servers/controllers of its configuration every 10 seconds as well.

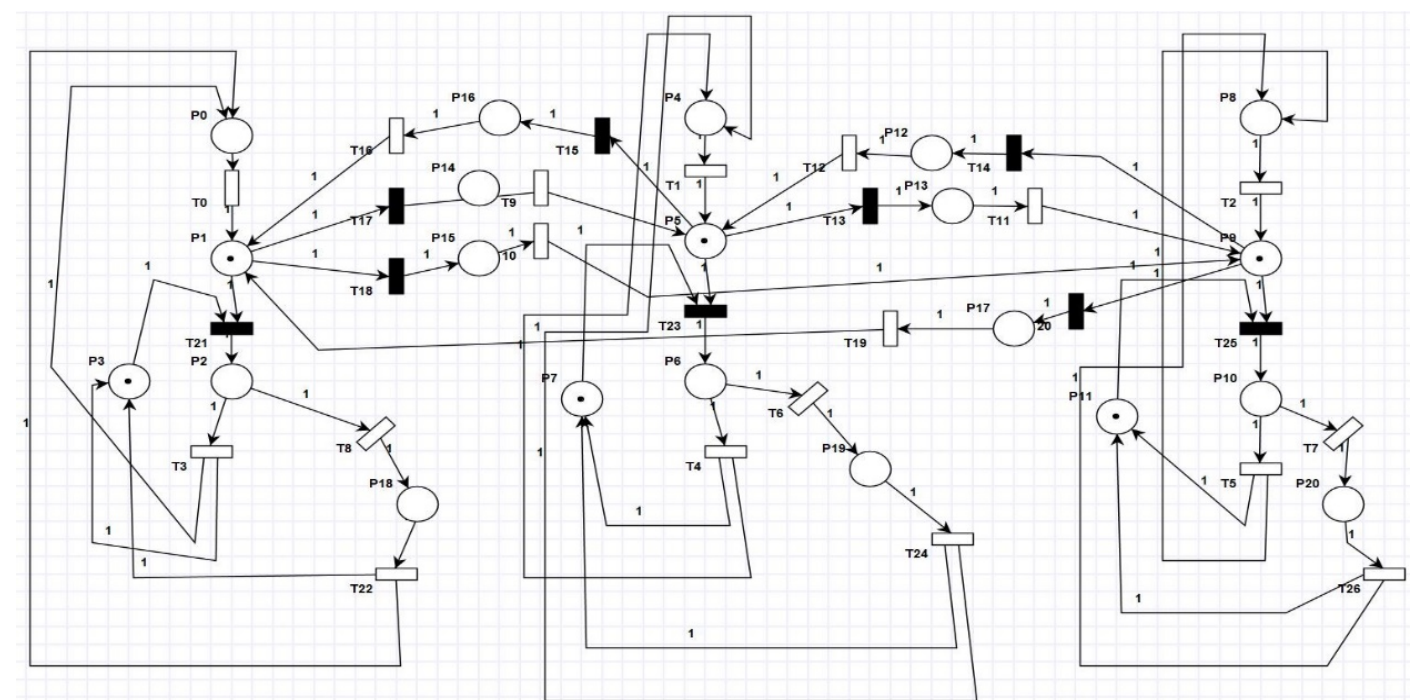

Figure 4. Proposed Parallel Topology for Software-Defined Networks.

Since all the three controllers represent a whole one main controller and the updates between the controlling entities will be every 10 seconds, that means that the configuration information will be merged together every 10 seconds, in other words the main triple-parts controller will update its general table of the whole network's status every 10 seconds. In case if there was a disruption on any one of the controllers, the other two controllers will fill the vacancy, left by the infected controller; and that's by serving the switches that were depending on the disrupted controller before it was disrupted. Of course; the switches will already be connected to all the three controllers so, in case of an attack; a bot that is already installed in the controllers; is going to be infiltrating the attacker's source IP. The attacked controller's IP will be isolated with the source IP of the attacker. Now all switches will be communicating with the controllers directly without any noticeable change, since that the remaining two controllers will add more space to deter Dos/DDoS attacks first and if this fails then the infected controller's IP will be isolated and the controllers continue their work like one controller of two main parts. The description of places is stated in the table 3.

Table 3

\begin{tabular}{ll} 
& Description of Places in Parallel Topology of SDN \\
\hline Place & Description \\
\hline P1/P5/P9 & Allocation of servers \\
\hline P2/P6/P10 & Server $1,2,3$ Active processing \\
\hline
\end{tabular}


Continuation Table 3

\begin{tabular}{ll}
\hline P12-P17 & $\begin{array}{l}\text { Exchanging the information and configuration } \\
\text { updates of the network }\end{array}$ \\
\hline P3/P7/P11 & $\begin{array}{l}\text { Recovery and restoring the working state of the } \\
\text { controller }\end{array}$ \\
\hline P18/P19/P20 & Attack on the server or controller \\
\hline P0/P4/P8 & Returning to the initial state \\
\hline
\end{tabular}

Description of transitions is in table 4.

Table 4

Description of Transitions in Parallel Topology of SDN

\begin{tabular}{ll}
\hline Transition & Description \\
\hline T21/T23/T25 & Server 1, 2, 3 Active processing \\
\hline T9-T20 & $\begin{array}{l}\text { Exchanging the updates of network configuration } \\
\text { between servers }\end{array}$ \\
\hline T3/T4/T5 & $\begin{array}{l}\text { Back to active processing/ processing the next } \\
\text { request }\end{array}$ \\
\hline T6/T7/T8 & Attack on server/controller \\
\hline T22/T24/T26 & Recovery from the attack \\
\hline T0/T1/T2 & Transition back to initial state \\
\hline
\end{tabular}

\section{Hybrid topology.}

The Hybrid topology contains six controlling nodes; three primary controllers and three spare ones as backup. Every main controller has a backup controller that could the next dominant primary controller in the situation of a disruption or a threat on its own current main controlling entity. As shown in design 5.

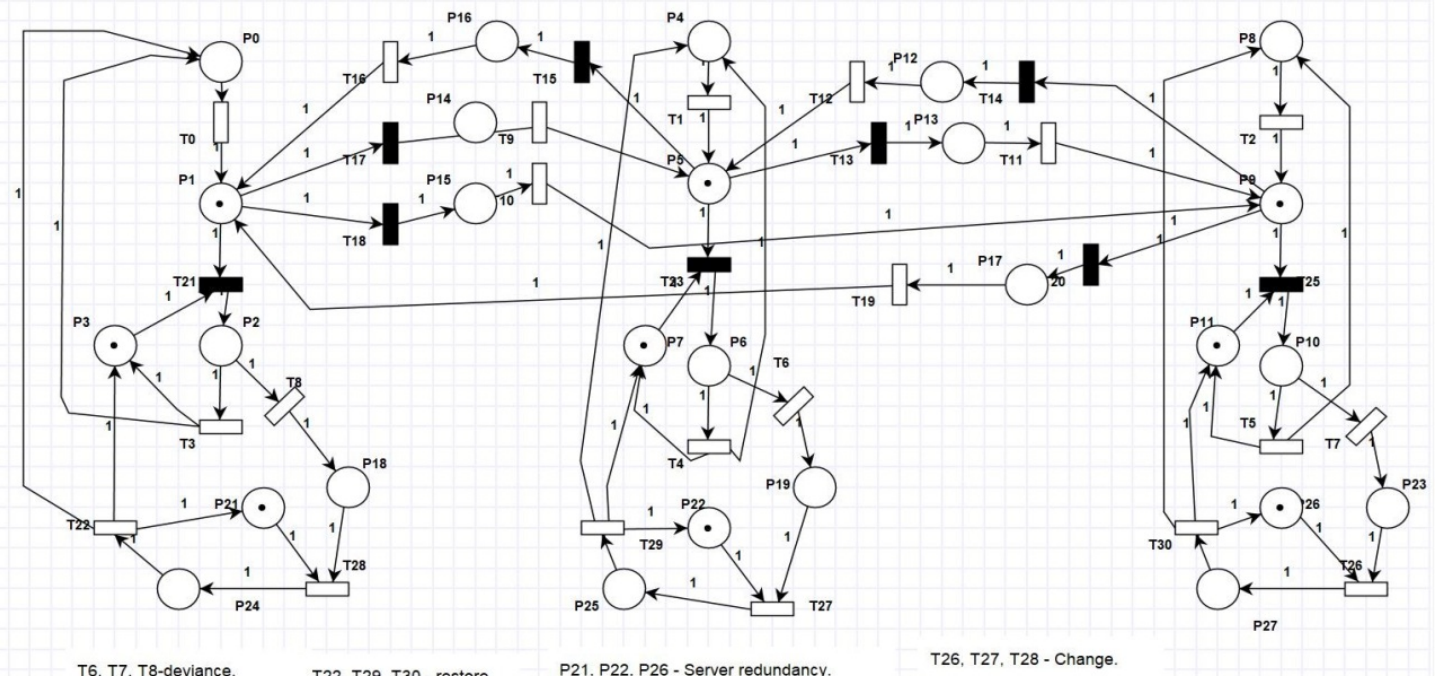

Figure 5. Proposed Hybrid Topology for Software-Defined Networks.

In addition, this topology can be considered as a mixture of the Serial and Parallel topologies, therefore it was named as Hybrid; the Hybrid topology's structure consists of six controlling nodes. Three of those six are main controllers working together in a parallel way as whole one brain and in this aspect this topology resembles the parallel topology therefore, 
in this situation; the same rules of parallel topology can be applied [8]. The rest of the six controllers which are three will be considered as a backup for the main controlling nodes meaning, one backup controller for every main controller. The backup controllers will be connected to other network nodes as well and that's done via two ways:

- Being in connection with its main primary controlling node to take its place in the cyberdisruption or attack situation.

- Also, every single back controller will have a connection channel with everyone of the other backup or spare controlling nodes.

In critical situations of a disruption on any of the three main control layer nodes; then it will be blocked by isolation alongside with the perpetrator's source IP and it will be replaced with its substitute until the compromised previously main controller is back to work. Not to forget that prior to conducting this process; there will be an embedment of a bot within the source address of the attacker. The table 5 describes the places of this diagram [8].

Table 5

\section{Description of Places in Hybrid Topology of SDN}

\begin{tabular}{ll}
\hline Place & Description \\
\hline P1/P5/P9 & Allocation of servers \\
\hline P21/P22/P26 & Extra servers / backup servers \\
\hline P2/P6/P10 & Active processing \\
\hline P18/P19/P23 & Server/controller under attack \\
\hline P24/P25/P27 & Recovery of server/controller \\
\hline P3/P7/P11 & Processing next request \\
\hline P0/P4/P8 & Returning to initial state \\
\hline P12-P17 & Exchanging the information across the network
\end{tabular}

The table 6 describes the transitions of the hybrid topology in the model of Petri Nets [8].

Table 6

\begin{tabular}{ll}
\multicolumn{2}{c}{ Description of Transitions in Hybrid Topology of SDN } \\
\hline Transition & Description \\
\hline T0/T1/T2 & Transition from initial state to active processing \\
\hline T21/T23/T25 & Active processing \\
\hline T3/T4/T5 & Processing next request \\
\hline T6/T7/T8 & Deviance or attack state \\
T22/T29/T30 & $\begin{array}{l}\text { Transitioning to backup /restoring/ back to initial } \\
\text { state }\end{array}$ \\
\hline T26/T27/T28 & Transformation and change \\
\hline T9-T20 & $\begin{array}{l}\text { Sharing and updating the network configuration } \\
\text { between servers }\end{array}$ \\
\hline
\end{tabular}

\section{Ordinary topology}

This modeling is conducted for representing the usual single-controller topology that has the name; the Ordinary topology in our research which has one controller and for describing its weakness points. 


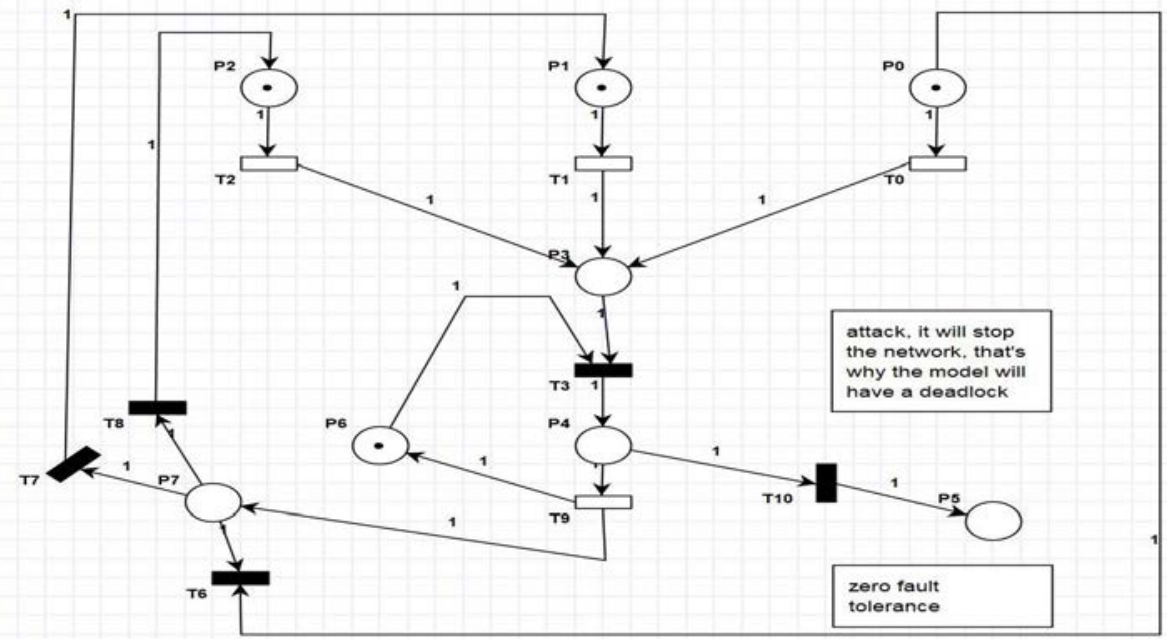

Figure 6. Proposed Ordinary Topology for Software-Defined Networks.

This topology describes a simple single-controller environment of Software-defined network. It is being mentioned and modelled here for comparative analysis purposes to depict the reliability of the presented framework alongside its suggested three environments [8]. This diagram describes the vulnerability of single-controller SDN environment and how it is attack-prone due to the single point of failure SPOF issue; that needs to be patched. This network is managed via a single controlling entity that deals with switches' requests as usual until an attack occurs. In the attack situation any kind of disruption, the aforementioned model proves that a simple attack focused on that only controller could jeopardize the whole network. That shows that this single-controller environment has a fault tolerance of zero value. The Table 7 depicts the single-controller diagram's places [6].

Table 7

\begin{tabular}{ll}
\multicolumn{2}{c}{ Places description in Ordinary Topology of SDN } \\
\hline Place & Description \\
\hline P0/P1/P2 & Selection of switches \\
\hline P3 & Main controlling node \\
\hline P4 & Active processing \\
\hline P6 & $\begin{array}{l}\text { Next request Processing / return to the } \\
\text { initial state }\end{array}$ \\
\hline P7 & Exchanging requests \\
\hline P5 & Compromise attempt on the controller
\end{tabular}

The table 8 describes transitions of Ordinary topology in a Petri Nets model [6].

Table 8

\begin{tabular}{ll}
\multicolumn{2}{c}{ Description of Transitions in Ordinary Topology of SDN } \\
\hline Transition & Description \\
\hline T0/T1/T2 & $\begin{array}{l}\text { Providing the controller with } \\
\text { requests }\end{array}$ \\
\hline T3 & Active processing \\
\hline T9 & Initial state/ dealing with switches \\
\hline T6/T7/T8 & Selection of switches \\
\hline T10 & Attack attempt \\
\hline
\end{tabular}


By conducting a simulation in the PIPE software using the GSPN module on the above models; it is possible to gain the tokens' average number in that lies in every controller to derive a mathematical relationship between the used topology's type and the average number of tokens that represent that requests dealt with by each place that represents a controller. In addition, it is obvious that the bigger the number of tokens or requests; the less the defense ability the controllers will have so that means that defense factor value has an opposite proportion to the number of tokens. One more thing to notice that the proposed mathematical relationship has to show the same results gained by the PIPE software which clearly state that the parallel topology is the best amongst all of the other topologies since it has the least average number of token/requests per unit of time and that what we have to model mathematically. Table 9 describes the tokens' average number in places/controllers in the models of the Petri Nets topologies [6], [8].

Table 9

\begin{tabular}{ccccc}
\multicolumn{4}{c}{ Average Tokens' number in SDN Controllers represented by PN Places in GSPN Module } \\
\hline Algorithms & $\begin{array}{c}\text { Serial } \\
\text { Topology }\end{array}$ & $\begin{array}{c}\text { Parallel } \\
\text { Topology }\end{array}$ & $\begin{array}{c}\text { Hybrid } \\
\text { Topology }\end{array}$ & $\begin{array}{c}\text { Ordinary } \\
\text { Topology }\end{array}$ \\
\hline P3 & 0.16337 & & $1.99975 \approx 2$ \\
\hline P7 & 0.06867 & & & \\
\hline P11 & 0.13233 & 0 & 0 & \\
\hline P1 & & 0 & 0 & \\
\hline P5 & & 0 & 0.9037 & \\
\hline P9 & & & 0.90368 \\
\hline P21 & & & 0.90352 \\
\hline P22 & &
\end{tabular}

The needed and created mathematical relationship is a relationship that could achieve the same results, as it will be shown afterwards. This relationship proved that based on the gained numerical results; it could be inferred that all the newly proposed three topologies work and behave better than the single-controller ordinary topology. Especially, the Parallel topology that is the ideal one theoretically and, from the mathematical relationship that was suggested in this research; we could acquire the same results and implications that match the acquired numerical results from the aforementioned simulation.

So, based on the simulation results, we propose an equation that represents the relationship between the number of controllers, type of interaction between them and the number of requests directed from/to them. The formula is derived to create a security factor that could be used a standard for determining the security intensity of any software-defined network that is leveraging any these three previously mentioned topologies [8]:

$$
\Delta=\sum_{\mathrm{i}=1}^{\mathrm{i}=\mathrm{n}} \mathrm{k}_{\mathrm{i}} \cdot \frac{1}{\sum_{\mathrm{z}=0}^{\mathrm{z}=\infty} \mathrm{z}_{\mathrm{i}}}
$$

Where $\mathrm{K}$ is the places number or amount, which describe the work of the controllers in every model, $\mathrm{Ki}=(\mathrm{K} 1, \mathrm{~K} 2 \ldots \mathrm{Kn})$ and $\mathrm{Z}$ represents the number value of tokens in those places $\mathrm{K}$ of 
those models. In addition, Zki $=(0 \ldots \infty)$. Of course, the Higher the value of the Defense Factor, the better it is. By applying the gained simulation results, it is possible to find that according to the law the best topology would be the $2^{\text {nd }}$ one or the parallel topology and that matches the simulation results shown in the table, that shows that the controllers in the $2^{\text {nd }}$ topology will be less occupied with requests than other topologies which means that they will be more available hence; have more reliability against Dos/DDoS attacks.

The article's scientific value focuses mainly on figuring a method to find a measurement for the security proportion or intensity of any network environment that leverages any one of the aforementioned topologies. The article here depicted those topologies briefly and compared between them and the Ordinary topology.

The proposed formula's reliability and correctness is inferred from its matching to the simulation gained results. Because both the acquired results and the formula's implementation prove and assure that all the suggested SDN controllers' topologies work in a better way than the ordinary one. In addition, those topologies' controllers are freer along the average time of processing meaning that; they are less busy than the controller of the ordinary topology which means that those controllers be more reliable and more deterring to attacks like DoS/DDoS which focus on submerging the controllers with pseudo requests.

\section{Conclusions}

We have presented in this article an idea about assuring the security of networks by assuring the security of software-defined networks hence, making it easier for classical structure networks to change to the SDN paradigm since it will be safer.

We discussed the main methods proposed to be integrated with the application layer as an application that will be a full framework to assure the security of SDN.

Alongside the proposed algorithms, here we explained briefly about the proposed working topologies in our research, that categorizes three main working topologies for softwaredefined networks' controllers.

Here we mainly focus on modelling the proposed topologies using Petri Nets system, to conduct a simulation on those modelled designs using PIPE software and to gain results that could show the best topology to be used with the emptiest space in its controllers; meaning the topology with the least vulnerable controllers to DoS/DDoS attacks. Based on the gained results we have created a mathematical relationship that will simulate the pattern of the gained results and their implications. This formula can be used as a theoretical mathematical instrument to figure out the cyber-threat level imposed on a network that leverages the SDN structure.

When we apply the acquired results from the simulation on this presented defense factor formula, the same implications could be gained. From the numerical simulation results, it is possible to conclude the following:

All the three proposed topologies are better than the usual one-controller SDN environment, which is the ordinary topology.

The parallel topology is the best of all four topologies since its controllers are emptier most of the average processing time.

And using the defense factor formula; it is possible to get a match for the gained numerical results which proves its feasibility.

The article's scientific value focuses mainly on figuring a method to find a measurement for the security deterrence level of any SDN that leverages any one of the 
aforementioned environments. This article depicted those topologies briefly and compared between them and the Ordinary topology.

The proposed formula's reliability and correctness is inferred from its matching to the simulation gained results. Because both the acquired results and the formula's implementation prove and assure that all the suggested SDN controllers' topologies work in a better way than the ordinary one. In addition, those topologies' controllers are freer along the average time of processing meaning that; they are less busy than the controller of the ordinary topology which means that those controllers be more reliable and more deterring against cyber-attacks which focus on submerging the controllers with pseudo requests.

\section{References}

1. Casado M. et. al., Ethane: Taking Control of the Enterprise. In: ACM SIGCOMM Computer Communication Review, 2007, 37(4), pp.1-12.

2. Shi L., LI Y., Feng H. Performance Analysis of Honeypot with Petri Nets. In: Information, 2018, 9(10), pp. 1-19.

3. Almutairi L., Shetty S. Generalized Stochastic Petri Net Model Based Security Risk Assessment of Software Defined Networks. In: Military Communications (MILCOM), 2017, pp. 545 - 550.

4. Zhou Y., Bai Y., Zhao P. A Petri Net-based Method to Verify the Security of the SDN. In: IOP Conference Series: Earth and Environmental Science, 2018, 234(1), pp. 1 - 6.

5. Prasad K., Reddy A., Rao K. DoS and DDoS Attacks: Defense, Detection and Trace Back Mechanisms - A Survey. In: Global journal of computer science and technology: E Network, Web and Security, Issue 7, 2014, JNTUH University, India. Volume 14, 18-pages.

6. Ameen A., Guţuleac E. Petri Nets Modeling for SDN topologies. In: Journal of Engineering Science, 2021, Technical University of Moldova, 28(2), pp.79-90.

7. Ameen A. Modelling sdn's parallel topology using petri nets. In: Journal of Engineering Science, (to be published).

8. Ameen A. Modelling proposed hybrid software-defined network controllers' topology using petri nets system. In: Studia Universitatis Moldaviae, 2021, Moldova State University, 2(142), pp.40-50.

9. Petri Net. [Online]. [accessed 05.01.2020]. Available: https://en.wikipedia.org/wiki/Petri_net.

10. Jyothi K., Dr. Reddy B., Study on Virtual Private Network (VPN), VPN's Protocols and Security. In: International Journal of Scientific Research in Computer Science, 2018, Sreenidhi Institute of Science and Technology, 3(5), pp. 919-932.

11. Ukwuoma H., Hammawa M., optimized key generation for RSA encryption: thesis. Ahmadu Bello University Zaria-Nigeria, 2015.

12. Ameen A. leveraging blockchain technology to assure security of SDN. In: Journal of Engineering Science, Proceedings of International conference on Electronics Communications and computing, October 2019, pages 128-139.

13. Puthal D., Movants S. P. Everything you wanted to know about Blockchain: its Promise, Components, Processes and Problems, Australia-USA, 2018.

14. Herweijer C., Waughray D., Warren S. Building Block (chain)s for a better planet, world economic forum, 2018.

15. Marconi foundation. (2018). Marconi Protocol: white paper. Retrieved 25.08.2019, from Marconi: https://docsend.com/view/5zragmb. 